\title{
RICHARD HENTSCHKE
}

DIE STELLUNG

DER VOREXILISCHEN SCHRIFTPROPHETEN ZUM KULTUS 

DIE STELLUNG

DER VOREXILISCHEN

SCHRIFTPROPHETEN

ZUM KULTUS

\author{
VON \\ R I CHARD HENTSCHKE
}

VERLAG ALFRED TÖPELMANN, BERLIN W 35 1957 


\section{BEIHEFTE ZUR ZEITSCHRIFT FUR DIE}

ALTTESTAMENTLICHE WISSENSCHAFT

75

Alle Rechte, einschl. der Rechte der Herstellung von Photokopien und Mikrofilmen von der Verlagshandlung vorbehalten

Printed in Germany

Satz: Walter de Gruyter \& Co., Berlin W 35

Druck: Buchkunst, Berlin W 35 
Diese Arbeit wurde von der Evangelisch-Theologischen Fakultät der Universität Münster als Inaugural-Dissertation angenommen. Referent war Prof. D. Dr. Wilhelm Rudolph und Korreferent Prof. D. Johannes Herrmann. Die mündliche Prüfung fand am 5. 12.1953 statt.

Hier möchte ich Herrn Prof. RudolpH für die wissenschaftliche Förderung und persönliche Hilfe, die er mir stets in väterlicher Weise zukommen lieB, besonders herzlich danken. Herrn Prof. D. Dr. JoH. Hempel bin ich für die Aufnahme dieser Arbeit in die Beihefte zur ZAW und für seine Hinweise bezüglich der notwendig gewordenen Kürzung sowie ihm und seiner Frau für das Mitlesen der Korrekturen zu tiefem Dank verpflichtet. 
